\title{
Bi-Objective Adaptive Large Neighborhood Search Algorithm for the Healthcare Waste Periodic Location Inventory Routing Problem
}

\author{
Ayyuce Aydemir-Karadag ${ }^{1}$ (D)
}

Received: 6 December 2020 / Accepted: 12 August 2021 / Published online: 18 September 2021

(c) King Fahd University of Petroleum \& Minerals 2021

\begin{abstract}
There has been an unexpected increase in the amount of healthcare waste during the COVID-19 pandemic. Managing healthcare waste is vital, as improper practices in the waste system can lead to the further spread of the virus. To develop effective and sustainable waste management systems, decisions in all processes from the source of the waste to its disposal should be evaluated together. Strategic decisions involve locating waste processing centers, while operational decisions deal with waste collection. Although the periodic collection of waste is used in practice, it has not been studied in the relevant literature. This paper integrates the periodic inventory routing problem with location decisions for designing healthcare waste management systems and presents a bi-objective mixed-integer nonlinear programming model that minimizes operating costs and risk simultaneously. Due to the complexity of the problem, a two-step approach is proposed. The first stage provides a mixed-integer linear model that generates visiting schedules to source nodes. The second stage offers a Bi-Objective Adaptive Large Neighborhood Search Algorithm (BOALNS) that processes the remaining decisions considered in the problem. The performance of the algorithm is tested on several hypothetical problem instances. Computational analyses are conducted by comparing BOALNS with its other two versions, Adaptive Large Neighborhood Search Algorithm and Bi-Objective Large Neighborhood Search Algorithm (BOLNS). The computational experiments demonstrate that our proposed algorithm is superior to these algorithms in several performance evaluation metrics. Also, it is observed that the adaptive search engine increases the capability of BOALNS to achieve high-quality Pareto-optimal solutions.
\end{abstract}

Keywords Healthcare waste $\cdot$ Location inventory routing $\cdot$ Periodic inventory routing $\cdot$ Bi-objective adaptive large neighborhood search

\section{List of symbols}

\section{Index set}

$Q \quad$ Set of treatment technologies, indexed by $q$

$T$ Set of treatment centers, indexed by $t$

$R$ Set of recycling centers, indexed by $r$

$F \quad$ Set of disposal centers, indexed by $f$

$S \quad$ Set of waste generation nodes, indexed by $s$

$D$ Set of central depots, indexed by $d$

$W$ Waste types, indexed by $w$

$P \quad$ Planning horizon, indexed by $p$

Ayyuce Aydemir-Karadag

aykaradag@cankaya.edu.tr

1 Department of Industrial Engineering, Faculty of Engineering, Cankaya University Main Campus, Yukariyurtcu Mah. Mimar Sinan Cad. No:4, 06790 Ankara, Turkey
$K \quad$ Fleet of collection vehicles, indexed by $k$

$i \quad$ Origin/destination, $i \in I=D \cup S \cup T \cup R \cup F$

$j \quad$ Origin/destination, $j \in J=S \cup T \cup R \cup F$ 


\begin{tabular}{|c|c|}
\hline$H C_{s}^{p}$ & $\begin{array}{l}\text { Inventory holding cost per period } p \text { for one unit } \\
\text { of waste at node } s\end{array}$ \\
\hline$M G_{s}^{w p}$ & Generated waste amount $w$ in period $p$ at node $s$ \\
\hline$A_{s}^{w}$ & $\begin{array}{l}\text { The amount of waste type } w \text { generated at node } s \\
\text { over the cyclic planning horizon; } \sum_{p} M G_{s}^{w p}\end{array}$ \\
\hline$S_{s}^{\prime}$ & Storage capacity of node $s$. \\
\hline$C A P V_{k}$ & Maximum capacity of a vehicle $k$ \\
\hline & Capacity of center $i, i \in\{T \cup R \cup F\}$ \\
\hline$R E_{w q}$ & $\begin{array}{l}\text { Mass reduction rate for waste type } w \text { by technol- } \\
\text { ogy } q\end{array}$ \\
\hline$R N_{r}$ & Mass reduction rate in recycling center $r$ \\
\hline$C O N$ & $\begin{array}{l}1 \text { If waste type } w \text { can be treated with treatment } \\
\text { technology } q\end{array}$ \\
\hline$k w$ & 1 If vehicle $k$ is compatible with waste type $w$ \\
\hline
\end{tabular}

\section{Decision variables}

$m_{r} \quad 1$ If a recycling center is installed at node $r$

$o_{f} \quad 1$ If a disposal center is installed at node $f$

$h_{q t} \quad 1$ If the treatment technology $q$ is installed at node $t$

$n_{q t}^{p} \quad 1$ If waste at location $t$ is treated with technology $q$ in period $p$

$x_{i j k}^{p} \quad 1$ If node $j$ is visited after node $i$ in period $p$ by vehicle $k$

$g_{s}^{p w} \quad 1$ If generation node $s$ is visited in period $p$ to collect waste type $w$

$e_{s k}^{p w} \quad$ Amount of waste type $w$ collected from generation node $s$ by vehicle $k$ in period $p$

$u_{w t}^{p} \quad$ Amount of waste type $w$ transported to treatment center $t$ in period $p$

$y_{t f w}^{p} \quad$ Amount of type $w$ waste residue directed from treatment center to disposal center $f$ in period $p$

$z_{r f}^{p} \quad$ Amount of waste residue directed from recycling center $r$ to disposal center $f$ in period $p$

$l_{i k}^{p} \quad$ The load of vehicle $k$ after visiting node $i$ in period $p$, $i \in\{S \cup T \cup R\}$

$I_{S}^{w p} \quad$ Inventory level of waste type $w$ at node $\mathrm{s}$ in period $p$

\section{Introduction}

Healthcare waste includes waste generated by different healthcare facilities. Hospitals generate $70 \%$ of the total healthcare waste. The remaining is generated by laboratories, research centers, mortuary and autopsy centers, animal research and testing laboratories, and blood banks. 75-95\% of total healthcare waste is classified as "non-hazardous", while the remaining 10-25\% is classified as "hazardous"[1]. Hazardous waste can pose serious environmental and health risks. Special care should be paid to treating this waste as it may contain infectious, pathological, pharmaceutical, cytotoxic, chemical, and radioactive substances. There are many technologies for disinfection of hazardous healthcare waste, such as pyrolysis, microwave, chemical or vaporized hydrogen peroxide application, dry heat, autoclave, $\mathrm{SF}-\mathrm{CO}_{2}$ sterilization, and radio-wave [2]. Sterilization and burn in incinerators at high temperatures have been widely used in practice [1-3]. Both methods effectively purify hazardous waste by removing all harmful effects of waste. Nonhazardous healthcare waste includes plastic water bottles, office papers, food waste, and packaging. This type of waste can be treated using technologies compatible with household waste. These technologies are recycling, biological reprocessing, waste-to-energy, incineration, bioremediation, and plasma gasification. Recycling is the most favorable option after reuse in the waste management hierarchy [3]. Therefore, it has priority over treatment. That is, if the waste cannot be safely recycled, it is diverted to the treatment. Such wastes are generally hazardous wastes containing chemicals or infectious substances.

During the COVID-19 pandemic, the need for medical services and high hospitalizations have resulted in a significant increase in healthcare waste $[4,5]$. This unexpected increase puts tremendous pressure on waste management systems to ensure that waste is collected, transported, and treated properly. Huge amounts of waste overwhelm the existing systems and require increasing the frequency of waste collection, the number of trucks assigned to collect COVID-19 waste from healthcare facilities and quarantine homes, and the waste processing capacity to treat those waste. Many countries (France, Netherlands, India, China, Iran, a few countries in Europe) have faced challenges when dealing with large volumes of waste [4]. Managing healthcare waste is vital for the world, as improper practices in the waste system can cause the further spread of the virus $[4,6,7]$.

In this paper, we address the periodic location inventory routing problem (PLIRP) for healthcare waste. This research is motivated by the challenges faced in managing healthcare waste during COVID-19. The problem includes the periodic collection of healthcare waste from waste generators and transporting them to recycling, treatment, and disposal centers. Thus, it creates cyclic visit schedules for healthcare facilities considering their inventory levels, determines the number of vehicles to be used each day, and the locations of waste processing centers. The problem tackles strategic and operational decisions in waste management systems simultaneously. Separating these decisions makes it difficult for decision makers to effectively establish and manage the waste systems while keeping economic, environmental, and risk factors to a minimum. Locating treatment centers account for a large part of the cost and risk in the system and depends mainly on routing decisions at the operational level. Periodic visits of vehicles to waste generation nodes prevent the accumulation of large amounts of waste in medical facilities, reducing the risk of transmission, explosion, and spread of 
the virus through waste. Waste-compatible vehicles must be used to collect waste and directed to centers equipped with waste-compatible technologies. Routing and inventory decisions are interrelated and affect shipping times and inventory levels at production nodes.

To the best of our knowledge, no study in the relevant waste literature has addressed PLIRP. The first contribution of this study is to introduce the problem to the literature and develop a bi-objective mathematical formulation. The objectives of the model are minimization of operating costs and total risk. The network design of the proposed problem allows the collection of hazardous and non-hazardous healthcare waste and the flow of waste between centers. Hazardous waste can be treated in incineration and sterilization centers known to kill the COVID-19 virus effectively. Non-hazardous types of waste can be either recycled or incinerated, and then, the waste residue is sent to landfilling (disposal centers). If recyclable waste can be efficiently segregated from other waste, it will significantly minimize waste, and that the whole waste management system will benefit from it.

Since the location routing problem is NP-hard [8], the extended variant of this problem adapted to the waste management system (WM) is also Np-hard. Some realistic restrictions specific to the WM problem, such as waste-towaste, waste-to-technology incompatibilities, complicate the problem further. The complexity of the problem has led the authors to develop a two-stage solution approach. The first stage presents a mixed-integer programming formulation that determines the collection schedules of healthcare facilities over the cyclic planning horizon. The second stage contributes to the literature by developing a Bi-Objective Adaptive Large Neighborhood Search Algorithm (BOALNS) to achieve high-quality Pareto-optimal solutions to the problems of vehicle routing, facility location, and transportation of waste and waste residues. We are not aware of any study that presents a multi-objective Adaptive Large Neighborhood Search Algorithm (MOALNS) in routing or location problems. Only one research in the literature [9] suggests MOALNS for solving the flow shop scheduling problem. However, we note that the Large Neighborhood Search Algorithm (LNS) and its variant (ALNS) have been successfully applied to various problems in the relevant area [10-17]. In LNS algorithms, destroy and repair operators are defined to explore a larger search space. ALNS differs from LNS in that the application of an operator in each iteration depends on its success during the search [18].

In the following, we review the existing literature. In Sect. 3, we define the problem and present the mathematical model for the proposed framework. The solution approaches are given in Sect. 4, and numerical results for several problem instances are provided in Sect. 5. Finally, Sect. 6 outlines the concluding remarks and presents future research directions.

\section{Literature Survey}

This section reviews the literature on location routing and periodic vehicle routing problems in healthcare and hazardous waste management and the most relevant studies on the periodic location routing problem. The literature search reveals that none of the relevant studies have concentrated on periodic routing integrated with location and inventory decisions in WM systems. Thus, there is a research gap in this regard.

A rich literature has emerged on location routing in waste management [19-32]. However, in all these papers, the waste is routed directly from waste generators to the storage or treatment center. Although there is no tour planning aspect, the addressed problem is called the location routing problem (LRP). For this reason, we do not elaborate on these studies but focus on the literature attempting to optimize decisions regarding vehicle routes and location selection simultaneously.

Zhao and Verter [33] consider a problem where oil is collected from generation nodes, stored at storage facilities, and shipped to integrated facilities. They determine the vehicle routes between generation nodes, facility locations, and plant capacity levels with minimum environmental risk and cost. Zhao and Ke [34] propose the first study combining inventory and location routing decisions in explosive waste management. They aim to minimize the inventory cost and the total inventory risk at collection centers. Rabbani et al. [35] develop two multi-objective evolutionary algorithms to route industrial waste with waste-compatible heterogeneous vehicles and locate hazardous waste centers. The objectives are to minimize total cost, transportation risk, and site risk. The performance of algorithms is tested on several problem instances. Later, Rabbani et al. [36] extend the problem by dealing with stochastic generation amounts at source nodes and inventory levels at treatment centers. A mixed-integer linear programming (MILP) model and a simulation-based approach are developed to solve the problem. Their model includes inventory costs associated with generation nodes in the objectives, but not inventory balance equations and inventory decisions in generation nodes. Multiple visits to generation nodes are not permitted, so there is no need to set visiting schedules. Farrokhi-Asl [37] focuses on collecting hazardous waste and selecting the locations for incineration, disposal, and recycling centers. They develop a Multi-Objective Hybrid Cultural And Genetic Algorithm to generate Pareto-optimal solutions to the problem. They contribute to the literature by incorporating into the model the fuel consumption and carbon dioxide emissions along the collection routes. Nikzamir and Baradaran [38] develop a mixed-integer nonlinear programming (MINLP) formulation for healthcare waste location and routing problem. The model includes stochastic transfer times between healthcare 
Table 1 Taxonomy of the most relevant literature

\begin{tabular}{|c|c|c|c|c|c|c|c|c|c|c|c|c|c|c|c|c|c|c|c|}
\hline \multirow[b]{2}{*}{ Study } & \multicolumn{4}{|c|}{$\begin{array}{l}\text { System Frame } \\
\text { work }^{\mathrm{a}}\end{array}$} & \multicolumn{6}{|c|}{ Decisions $^{\mathrm{b}}$} & \multicolumn{2}{|l|}{ Compatibility } & \multicolumn{5}{|c|}{ Objectives $^{\mathrm{c}}$} & \multirow[t]{2}{*}{ Multi-period } & \multirow[t]{2}{*}{ Waste type $^{\mathrm{d}}$} \\
\hline & $\mathrm{T}$ & $\mathrm{R}$ & $\mathrm{D}$ & S & $\mathrm{P}$ & TW & $\mathrm{L}$ & A & $\mathrm{R}$ & I & Waste-waste & $\begin{array}{l}\text { Waste- } \\
\text { Tecnology }\end{array}$ & $\mathrm{C}$ & $\mathrm{R}$ & $\mathrm{T}$ & $\mathrm{E}$ & $\mathrm{M}$ & & \\
\hline $\begin{array}{l}\text { Zhao and } \\
\text { Verter [33] }\end{array}$ & $\mathrm{X}$ & & $X$ & $\mathrm{X}$ & & & $X$ & $X$ & $X$ & & & $X$ & $\mathrm{X}$ & $X$ & & & & & $\mathrm{O}$ \\
\hline $\begin{array}{l}\text { Zhao and } \mathrm{Ke} \\
\text { [34] }\end{array}$ & & $X$ & & $\mathrm{X}$ & & & $X$ & $X$ & $X$ & $\mathrm{X}$ & & & $X$ & $X$ & & & & & $\mathrm{H}$ \\
\hline $\begin{array}{l}\text { Rabbani et al. } \\
\text { [35] }\end{array}$ & $X$ & $X$ & $X$ & & & & $X$ & $X$ & $X$ & $\mathrm{X}$ & $\mathrm{X}$ & $X$ & $\mathrm{X}$ & $\mathrm{X}$ & & & & & $\mathrm{H}$ \\
\hline $\begin{array}{l}\text { Rabbani et al. } \\
\text { [36] }\end{array}$ & $\mathrm{X}$ & $\mathrm{X}$ & $\mathrm{X}$ & & & & $\mathrm{X}$ & $\mathrm{X}$ & $X$ & & $X$ & $\mathrm{X}$ & $X$ & $X$ & & & & $\mathrm{X}$ & $\mathrm{H}$ \\
\hline $\begin{array}{l}\text { Farrokhi-Asl } \\
\text { et al. [37] }\end{array}$ & $\mathrm{X}$ & $\mathrm{X}$ & $X$ & & & & $\mathrm{X}$ & $\mathrm{X}$ & $X$ & & $\mathrm{X}$ & $\mathrm{X}$ & $\mathrm{X}$ & $X$ & & $\mathrm{X}$ & & & M \\
\hline $\begin{array}{l}\text { Nikzamir and } \\
\text { Baradaran } \\
{[38]}\end{array}$ & $\mathrm{X}$ & $\mathrm{X}$ & $X$ & & & $\mathrm{X}$ & $\mathrm{X}$ & $\mathrm{X}$ & $X$ & & $\mathrm{X}$ & $X$ & $\mathrm{X}$ & & & & $\mathrm{X}$ & & M \\
\hline $\begin{array}{l}\text { Tirkolaee } \\
\text { et al. [39] }\end{array}$ & & & $\mathrm{X}$ & & & $\mathrm{X}$ & $\mathrm{X}$ & $\mathrm{X}$ & $X$ & & & & & $X$ & $X$ & & & $\mathrm{X}$ & M \\
\hline This study & $\mathrm{X}$ & $\mathrm{X}$ & $\mathrm{X}$ & & $\mathrm{X}$ & & $\mathrm{X}$ & $\mathrm{X}$ & $\mathrm{X}$ & $\mathrm{X}$ & $\mathrm{X}$ & $\mathrm{X}$ & $\mathrm{X}$ & $\mathrm{X}$ & & & & $\mathrm{X}$ & M \\
\hline
\end{tabular}

and treatment centers and emission of contamination. $\mathrm{A} \mathrm{Bi}$ Objective Water-Flow Like Algorithm is proposed, which concurrently aims to minimize the total cost and emission of contamination. Tirkolaee et al. [39] offer a decision support system (DSS) to manage medical waste in the COVID19 pandemic. The proposed DSS includes developing a MILP model for the multi-trip location routing problem with time windows, a fuzzy chance-constrained programming approach to handle the uncertain demand in waste generation nodes, and a weighted goal programming approach. The formulation allows the vehicles to start and end the routes at different disposal centers and minimizes the total traveling time, the total violation from time windows, and site risk at disposal centers. Table 1 summarizes some key features of the reviewed studies in healthcare and hazardous waste location routing problems.

The periodic vehicle routing problem (PVRP) finds the visit schedule to each customer, assuming that customers have fixed service frequencies and a fixed quantity is delivered at each visit [40]. PVRP was firstly studied by Beltrami and Bodin [41] on municipal waste collection. Later, Russell and Igo [42] defined the problem, and Christofides and Beasley [43] formulated the mathematical model for PVRP. If inventory decisions are integrated into the PVRP, the problem is called the inventory routing problem (IRP). Unlike PVRP, IRP allows multiple visits to customers with variable delivery quantities. The inventory level is calculated at each time period considering the customer's daily product consumption rates [44]. Different variants of IRP have been studied in the relevant literature [45-48]. Few papers address periodic inventory routing in the context of waste management $[10,11,49,50]$. The former three studies focus on selective and periodic inventory routing problem (SPIRP), which differs from the classic IRP, by allowing some oil waste generation nodes not to be visited at all over the planning horizon. Aksen et al. [49] formulate two different MILP models capable of selecting and routing customers and determining purchasing decisions. The formulations are limited to solve only small-sized problems with 25 source nodes. Later, an adaptive large neighborhood search algorithm (ALNS) is developed by Aksen et al. [10] to obtain solutions for problems of up to 100 nodes. A heuristic algorithm that effectively solves PVRP up to 3000 nodes is provided by CardenasBarron et al. [11]. Taslimi et al. [50] deal with the periodic collection of medical waste and the inventory decisions at waste generators. They develop a decomposition-based heuristic algorithm where a column generation approach is used to solve each subproblem. The proposed problem allows not to visit some medical centers in one or more periods and incorporates transportation risk and occupational risk at healthcare centers.

The periodic location routing problem (PLRP) extends IRP by including location decisions. PLRP was first studied by Prodhon [51]. The author later improves her first 
approach by developing a hybrid evolutionary algorithm that outperforms the previous algorithms developed for PLRP [52]. Hemmelmayr [14] presents sequential and parallel large neighborhood search algorithms to find new best-known solutions. This study reveals that parallel versions perform better than sequential versions. Koç [17] integrates periodic routing of heterogeneous vehicles and facility location decisions, considering time windows while serving customers. In this study, a Unified Adaptive Large Neighborhood Search Algorithm (U-ALNS) is proposed, which outperforms previous algorithms in the literature. In PLRP or its variants, the locations of the depots where the vehicles start and finish their routes are determined. However, in WM systems, the problem involves locating waste processing centers but not depots. In addition, the transportation problem for waste residues between centers needs to be solved.

The preceding discussion on the relevant literature reveals that no studies address and solve PLIRP for WM systems. This paper concentrates on PLIRP to fill the gap in the literature and presents a bi-objective mixed-integer nonlinear programming formulation of the problem. The model is complicated as it incorporates numerous real-life aspects from the WM systems, such as waste classes, compatibility issues for wastes, vehicles, and technologies, and periodic visits to generation nodes. Since the formulation is nonlinear and the problem is Np-hard, we propose a two-stage solution approach. The first stage presents a mixed-integer linear formulation of the problem that generates visiting schedules of source nodes. The second stage provides a Bi-objective Adaptive Large Neighborhood Search Algorithm (BOALNS) that deals with vehicle routing, locations of centers, and transportation of waste and waste residues. BOALNS enables us to find high-quality Pareto-optimal solutions in a reasonably short time for the problems with up to 100 waste generation nodes and 58 waste centers.

\section{Problem Description and Formulation}

In the proposed problem, healthcare facilities are waste generation nodes. They create two types of healthcare waste: non-hazardous and hazardous. Waste producers are responsible for separating wastes according to their types. Heterogeneous vehicles start their trip from a central depot, visit the waste generators and collect vehicle-compatible waste. Vehicles have limited capacities, and each vehicle is compatible with only one type of waste. After collection, vehicles unload their waste into waste-compatible centers. When the vehicle carries hazardous healthcare waste, it can empty its load to a treatment center. A treatment center can be equipped with either one of the following technologies: sterilization and incineration. Since both technologies kill the coronavirus, they can be used safely for treating haz- ardous healthcare waste. The ash (waste residue) generated as a result of the incineration process is sent to the disposal center. It is assumed that there is no waste residue at the end of the sterilization process. If a vehicle carries non-hazardous waste, it can drop off its load into a treatment center equipped with incineration technology or a recycling center. Recycled waste residue and ash are sent to disposal centers. After a vehicle empties its load at one of the recycling, incineration, or sterilization centers, it ends its route at the central depot. A transportation network is created to transfer waste residues from incineration and recycling centers to disposal centers. The waste processing centers have limited capacities.

Periodic waste collection from healthcare facilities is allowed; thus, a cyclic weekly routing schedule is generated that is divided into five days. A healthcare facility can be visited several times during a cycle. Considering that each vehicle is compatible with a single type of waste, to collect all kinds of waste generated by a healthcare facility, the healthcare facility must be visited in a day by as many vehicles as the number of waste types it generates. The entire amount of vehicle-compatible waste accumulated in a healthcare facility must be collected during the visit of the vehicle; that is, partial waste collection is not allowed. If the healthcare facility is not visited by a vehicle compatible with the type of waste it produces, it accumulates the waste in the storage. Each healthcare facility has a limited storage capacity. Therefore, the total accumulated amount of waste in a healthcare facility cannot be more than its storage capacity. The network representation of the proposed system is illustrated in Fig. 1. It shows the decision variables (in rectangles and circles) related to the locations of centers and the amount of flow from nodes to centers.

The proposed mathematical formulation of the problem determines (1) visiting schedules to satisfy the periodic waste collection of source nodes, (2) the set of routes to be performed, (3) the number of vehicles to be used, (4) the inventory level at each source node, (5) the locations of centers, and (6) amount of waste residues transported between recycling, treatment and disposal centers. The objectives are to minimize operating costs and the total risk associated with transportation and site risks.

The assumptions in the formulation are as follows: (1) All of the parameters are deterministic. (2) There is no working inventory in waste generation nodes at the beginning or end of the cyclic planning horizon. (3) Only one type of technology can be installed in treatment centers. (4) Each vehicle is compatible with only one type of waste. (5) During a vehicle's visit, all vehicle-compatible waste accumulated in a generation node must be collected. The proposed formulation is not limited to model hazardous and non-hazardous healthcare waste. It can also develop a waste collection and treatment network for different types of waste where the same waste treatment and disposal processes are carried out. However, 
Fig. 1 The framework of the proposed waste management system

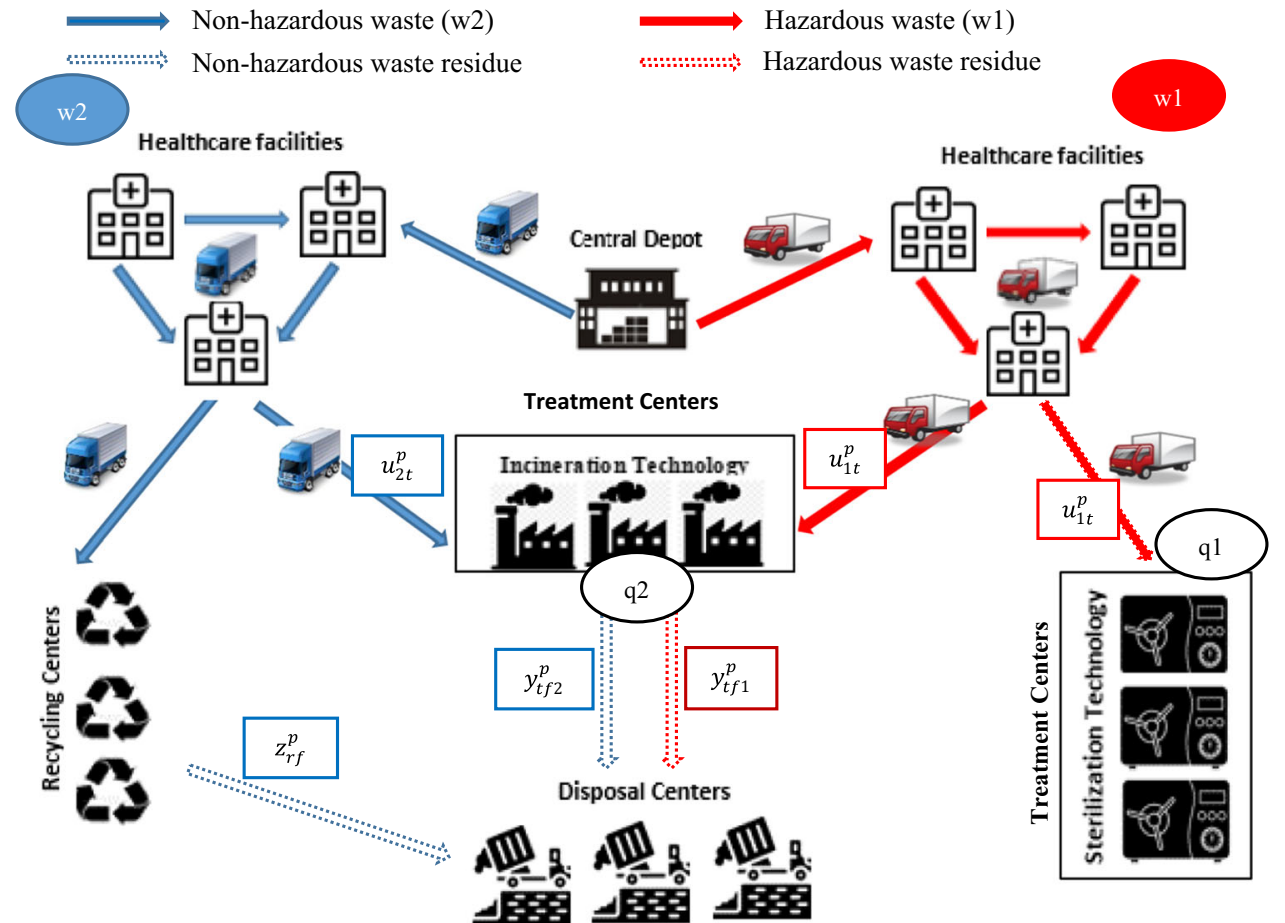

if new waste streams (e.g., from treatment to recycling centers) are introduced, the model should be expanded to include them.

The bi-objective mixed-integer nonlinear formulation of the PLIRP is as follows:

$$
\begin{aligned}
\operatorname{Min} f_{1}(x)= & \sum_{i \in S} \sum_{j \in S \cup T \cup R} \sum_{k \in K} \sum_{p \in P} C_{i j} x_{i j k}^{p} l_{j k}^{p} \\
& +\sum_{t \in T} \sum_{f \in F} \sum_{p \in P} \sum_{w \in W} C_{t f} y_{t f w}^{p} \\
& +\sum_{r \in R} \sum_{f \in F} \sum_{p \in P} C_{r f} z_{r f}^{p}+\sum_{t \in T} \sum_{q \in Q} E C_{t q} h_{q t} \\
& +\sum_{r \in R} E C_{r}^{\prime} m_{r}+\sum_{f \in F} E C_{f}^{\prime} o f \\
& +\sum_{t \in T} \sum_{w \in W} \sum_{p \in P} \sum_{q \in Q} u_{w t}^{p} n_{q t}^{p} U C_{q t} \\
& +\sum_{r \in R} \sum_{p \in P} \sum_{k \in K} \sum_{s \in S} x_{s r k}^{p} l_{s k}^{p} V_{k w=2} U C_{r}^{\prime} \\
& +\sum_{f \in F} \sum_{p \in P}\left(\sum_{r \in R} z_{r f}^{p}+\sum_{w \in W} \sum_{t \in T} y_{t f w}^{p}\right) U C_{f}^{\prime} \\
& +\sum_{s \in S} \sum_{w \in W} \sum_{p \in P} I_{s}^{w p} H C_{s}^{p}
\end{aligned}
$$

Equation 1 minimizes operating costs, including transportation costs between nodes, fixed location and variable costs at centers, and total inventory holding cost at source nodes.

$$
\begin{aligned}
\operatorname{Min} f_{2}(x)= & \sum_{t \in T} \sum_{w \in W} \sum_{p \in P} \sum_{q \in Q} P A_{t} u_{w t}^{p} h_{q t} \\
& +\sum_{i \in S} \sum_{j \in S \cup T U} \sum_{k \in K} \sum_{p \in P} P O P_{i j} x_{i j k}^{p} l_{i k}^{p}
\end{aligned}
$$

Healthcare waste may contain viable COVID-19 virus and possibly be a source of infection, so reducing risk is significant to prevent virus contamination through waste. Equation 2 minimizes the sum of the site and transportation risk. The first term includes the site risk at treatment centers equipped with incineration and sterilization technologies. Site risk is related to the amount of waste processed at these centers and population exposure around these centers. The second term is associated with the transportation risk. The total transportation risk is related to the amount of waste transported between source nodes, from source nodes to centers, and the number of people living along the route. We assume that waste residues do not pose any risk.

Constraints (3) and (4) allow multiple visits of vehicles to generation node $s$ in period $p$ to collect different types of wastes. They ensure that if a vehicle enters a node, it must also leave the node to another destination.

$$
\sum_{k \in K} \sum_{j \in S \cup R \cup T} x_{s j k}^{p} V_{k w}=g_{s}^{p w}, \quad \forall s \in S, p \in P, w \in W
$$


$\sum_{k \in K} \sum_{j \in D \cup S} x_{j s k}^{p} V_{k w}=g_{s}^{p w}, \quad \forall s \in S, p \in P, \mathrm{w} \in W$

Constraints (5) indicate that incoming and outgoing degrees of a depot are equal in period $p$.

$\sum_{j \in R \cup T} x_{j d k}^{p}-\sum_{s \in S} x_{d s k}^{p}=0, \quad \forall d \in D, k \in K, p \in P$

Constraints (6) determine the number of vehicles leaving the depot $d$ in period $p$ to collect waste type $w$.

$C A P V_{k} V_{k w} \sum_{s \in S} x_{d s k}^{p} \geq \sum_{s \in S} e_{s k}^{w p}, \quad \forall k \in K, p \in P, d \in D, w \in W$

Constraints (7) determine the inventory level of waste type $w$ at each generation node $s$ in time period $p$. The amount of end-of-period inventory for waste type $w$ equals the inventory transferred from the previous period plus the amount of waste type $w$ generated in period $p$ minus the total amount of waste type $w$ collected by all vehicles in period $p$.

$I_{s}^{w p}=I_{s}^{w p-1}+M G_{s}^{w p}-\sum_{k \in K} e_{s k}^{w p} V_{k w}, \quad \forall s \in S, p \in P, w \in W$

Constraints (8) indicate that the inventory level is zero at the beginning and end of the cyclical planning horizon.

$I_{s}^{w 0}+I_{s}^{\mathrm{wmax}\{P\}}=0, \quad \forall s \in S, w \in W$

Constraints (9) ensure that partial collection of waste is not allowed. If the generation node $s$ is visited in period $p$ to collect waste type $w$, the whole amount accumulated from this type of waste must be collected. Thus, the end-of-period inventory level is zero. Otherwise, the inventory level in generation node $s$ is at most $A_{s}^{w}$.

$I_{s}^{w p} \leq A_{s}^{w}\left(1-g_{s}^{p w}\right), \quad \forall s \in S, p \in P, w \in W$

Constraints (10) set an upper limit for the amount of waste collected from each generation node. The amount collected from generation node $s$ cannot exceed the storage capacity of node $s$.

$\sum_{k \in K} \sum_{w \in W} e_{s k}^{w p} \leq S_{s}^{\prime}, \quad \forall s \in S, p \in P$

Constraints (11) determine the load of the vehicle $k$ after it leaves the generation node $s$, considering each vehicle is compatible with only one type of waste. Constraints (12) and (13) control the upper limits of a vehicle's load, while Constraints (14) control the lower limits.

$l_{s k}^{p}-l_{j k}^{p}=e_{s k}^{p w}, \quad \forall s \in S, j \in\{S \cup D\}, k \in K, p \in P, w \in W$

$$
\begin{aligned}
l_{j k}^{p} & \leq \sum_{w \in W} x_{s j k}^{p} V_{k w}\left(C A P V_{k}-M G_{j}^{w p}\right), \quad \forall s \\
& \in\{S \cup D\}, j \in S, k \in K, p \in P \\
l_{j k}^{p} & \leq C A P V_{k}-e_{j k}^{p w}, \quad \forall j \in S, k \in K, p \in P, w \in W \\
l_{j k}^{p} & \geq \sum_{w \in W} V_{k w}\left(e_{j k}^{p w}-A_{j}^{w}\left(1-x_{s j k}^{p}\right)\right), \quad \forall j, s \in S, k \in K, p \in P
\end{aligned}
$$

Constraints (15) require the vehicle to have a zero load after leaving its waste at a treatment or recycling center.

$l_{j k}^{p}=0, \quad \forall j \in\{T \cup R\}, k \in K, p \in P$

Constraints (16) and (17) ensure that wastes are directed to centers equipped with appropriate technologies. Constraints (16) satisfy that a vehicle loaded with hazardous waste ( $w$ $=1$ ) can empty its load to a treatment center equipped with sterilization $(q=1)$ or incineration technologies $(q=2)$. On the other hand, a vehicle carrying non-hazardous waste ( $w$ $=2$ ) may leave its waste at a treatment center with incineration technology according to Constraints (16) or a recycling center according to Constraints (17).

$x_{s t k}^{p} \leq \sum_{w \in W} \sum_{q \in Q} V_{k w} . C O M_{w q} h_{q t}, \quad \forall k \in K, s \in S, t \in T, p \in P$

For $w=1 q=1,2$

For $w=2 q=2$

$x_{s r k}^{p} \leq V_{k w=2} m_{r}, \quad \forall k \in K, s \in S, r \in R, p \in P$

The assumption that only one type of technology can be installed in each treatment center is provided by Constraints (18).

$\sum_{q \in Q} h_{q t} \leq 1, \quad \forall t \in T$

Constraints (19) ensure that if technology $q$ is installed in treatment center $t$, waste can be treated with technology $q$ in period $p$ in that center.

$n_{q t}^{p} \leq h_{q t}, \quad \forall t \in T, q \in Q, p \in P$

Constraints (20) specify the amount of waste type $w$ directed from generation nodes to treatment centers.

$u_{w t}^{p}=\sum_{k \in K} \sum_{s \in S} x_{s t k}^{p} l_{s k}^{p} V_{k w}, \quad \forall t \in T, p \in P, w \in W$ 
Constraints (21) and (22) ensure that the amount of waste residue generated in treatment and recycling centers is equal to the amount of waste reduced by the mass loss rate at each center.

$\sum_{f \in F} y_{t f w}^{p}=u_{w t}^{p} n_{q t}^{p}\left(1-R E_{w q}\right), \quad \forall p \in P, t \in T, w \in W$

$\sum_{f \in F} z_{r f}^{p}=\left(1-R N_{r}\right) \sum_{k \in K} \sum_{s \in S} x_{s r k}^{p} l_{s k}^{p} V_{k w=2}, \quad \forall r \in R, p \in P$

Constraints (23-25) indicate that the capacity of the centers cannot be exceeded.

$$
\begin{aligned}
& \sum_{w \in W} u_{w t}^{p} \leq \sum_{q \in Q} C A P_{t} h_{q t}, \quad \forall t \in T, p \in P \\
& \sum_{k \in K} \sum_{s \in S} x_{s r k}^{p} l_{s k}^{p} V_{k w=2} \leq C A P_{r} m_{r}, \quad \forall r \in R, p \in P \\
& \sum_{r \in R} z_{r f}^{p}+\sum_{w \in W} \sum_{t \in T} y_{t f w}^{p} \leq C A P_{f} o_{f}, \quad \forall f \in F, p \in P
\end{aligned}
$$

Constraints (26-27) are the non-negativity and binary constraints.

$$
\begin{aligned}
& e_{s k}^{p w} \geq 0, \quad \forall s \in S, w \in W, p \in P, k \in K \\
& u_{w t}^{p} \geq 0, \quad \forall t \in T \quad p \in P, w \in W \\
& y_{t f w}^{p} \geq 0, \quad \forall t \in T, f \in F, p \in P, w \in W \\
& z_{r f}^{p} \geq 0, \quad \forall r \in R, \quad f \in F, p \in P \\
& l_{i k}^{p} \geq 0, \quad \forall i \in\{S \cup T \cup R\}, k \in K, p \in P \\
& I_{s}^{w p} \geq 0, \quad \forall s \in S, p \in P, w \in W \\
& m_{r} \in\{0,1\}, \quad \forall r \in R \\
& o_{f} \in\{0,1\}, \quad \forall f \in F \\
& h_{q t} \in\{0,1\}, \quad \forall t \in T, q \in Q \\
& n_{q t}^{p} \in\{0,1\}, \quad \forall t \in T, q \in Q, p \in P \\
& x_{i j k}^{p} \in\{0,1\}, \quad \forall i, j \in\{D \cup S \cup R \cup T\}, k \in K, p \in P \\
& g_{s}^{p w} \in\{0,1\}, \quad \forall i, j \in\{D \cup S \cup R \cup T\}, k \in K, p \in P
\end{aligned}
$$

\section{Solution Methodology}

Since PLIRP is NP-hard, we propose a two-stage solution methodology. The first stage, called the pick-up plan generation (PP), presents a mixed-integer linear program that determines the collection schedules of healthcare facilities over the cyclic planning horizon. The second stage provides BOALNS, which generates a solution to the problem using the information obtained in the first stage. The details of the solution methods are explained in the following sub-sections.

\subsection{Pick-up Plan Generation}

PP finds the visit periods to generation nodes, the amount of waste collected from each node at each visit, and the inventory levels at source nodes, taking into account their storage capacities. In addition, vehicles are assigned to generation nodes considering their waste-compatible capacities. $\mathrm{PP}$ formulation requires the use of additional parameters and decision variables given below.

Additional parameters:

\begin{tabular}{ll}
\hline$C_{s p}$ & Average routing cost for visiting generation node s in period $\mathrm{p}$ \\
$C O$ & Operating cost per vehicle \\
$H$ & Inventory holding cost per period per unit of waste in the \\
& source node
\end{tabular}

Additional decision variables:

$\rho_{k p}$

Number of vehicles $k$ used in period $p$

$\operatorname{Min} \sum_{s \in S} \sum_{p \in P} \sum_{w \in W} C_{s p} g_{s}^{p w}$
$+C O \sum_{k \in K} \sum_{p \in P} \rho_{k p}+H \sum_{s \in S} \sum_{p \in P} \sum_{w \in W} I_{s}^{w p}$

$C A P V_{k} \rho_{k p} \geq \sum_{w \in W} \sum_{s \in S} e_{s k}^{p w} V_{k w}, \quad \forall p \in P, k \in K$

$e_{s k}^{p w} \geq 0, \quad \forall s \in S, w \in W, p \in P, k \in K$

$\rho_{k p} \geq 0, \quad \forall p \in P, k \in K$

$I_{s}^{w p} \geq 0, \quad \forall s \in S, w \in W, p \in P$

$g_{s}^{p w} \in\{0,1\}, \quad \forall s \in S, p \in P, w \in W$

and (7-10) from the PLIRP formulation.

The objective function in Eq. (28) minimizes the total approximated routing cost, vehicle operating, and inventory holding costs. Constraints (29) determine the number of vehicles dispatched to collect waste accumulated in generation 
nodes in period $p$. We note again that each vehicle is compatible with a single type of waste. Constraints (30-33) are non-negativity and binary integrality constraints.

\subsection{Bi-objective Adaptive Large Neighborhood Search Algorithm}

The second stage presents a BOALNS algorithm to determine collection routes of vehicles and locations of centers with minimum total cost and risk. It also solves the problem arising in the transportation of waste residues between centers. The algorithm is designed to generate Pareto-optimal solutions for the bi-objective optimization of the proposed problem.

We used a total of ten destroy and insertion operators to change the center configurations, inter-period, and intraperiod routes. Figure 2 provides the details of the BOALNS. The search process consists of several segments, each with the same number of $\varphi$ iterations $[10,12,18]$. At each iteration, a destroy and an insertion operator are applied successively to the current solution to generate a neighboring solution. The operators are selected using the roulette wheel mechanism principle. Each operator has weights associated with its performance in terms of both objective functions in the last segment. Let $w_{p k}$ be the weight of operator $p$ in terms of objective function $k$, then its selection probability is $w_{p k} / \sum_{i=1}^{10} w_{i k}$. The performance of an operator is scored. The higher the operator's score, the better solutions it generates. If the neighboring solution $\left(s^{\prime}\right)$ obtained with the operator dominates some solutions in the Pareto-optimal set (POS), the operator's score is increased by $\sigma_{1}$ (Rows 10-14 in Fig. 2) and the dominated solutions are removed from POS. If it generates a non-dominated solution, its score is increased by $\sigma_{2}$ (Rows $15-18$ in Fig. 2). In both cases, the neighboring solution is accepted as the current solution, $s$ $\leftarrow s^{\prime}$. However, if a poor quality solution is generated, it is compared to the current solution in terms of a single objective function selected using the multinomial probability mass function. This approach has been successfully applied in several multi-objective metaheuristics [53, 54]. Since there are two objectives in our problem, we give an equal probability of selection $(50 \%)$ to both. If the operator improves the solution for the selected objective, it is accepted as the current solution, $s \leftarrow s$, and the operator's score is increased by $\sigma_{3}$ (Rows 22-24 in Fig. 2). Otherwise, it is evaluated by the simulated annealing criterion, and the score of the operator is not changed. The selection probability for simulated annealing criterion is calculated by $e^{-\left(f\left(s^{\prime}\right)-f(s)\right) / T}$. In the beginning, we initialize a temperature $T^{\text {start }}$, where it is gradually decreased at each iteration by a cooling factor $\alpha$.

In the beginning, each operator has the same selection probability. The scores are set to 0 . We update the weights of operators $\left(w_{i k j}\right)$ at the end of each segment with Eq. (34), which increases the selection probability of operators with higher scores.

$w_{i, k, j+1}=\left\{\begin{array}{l}w_{i k j} i f o_{i j}=0 \\ (1-\eta) w_{i k j}+\frac{\eta \pi_{i k}}{o_{i j}} \text { ifo } o_{i j} \neq 0\end{array}\right.$

where $w_{i, k, j+1}$ represents the weight of operator $i$ for objective function $k$ to be used in segment $j+1 ; \pi_{i k}$ is the score of operator $i$ for objective function $k ; o_{i j}$ indicates the frequency of using operator $i$ in segment $j ; \eta$ is a factor between 0 and 1 that controls the effect of the weight changes on operator performance.

\subsubsection{Initialization}

The solution produced by PP is used to generate an initial solution to the BOALNS. The visited nodes where the same type of waste is collected on the same day are grouped. A visit sequence among these generation nodes is constructed using a greedy insertion operator (see Sect. 4.2.2). Each vehicle is then directed to the waste-compatible centers. We note that non-hazardous wastes are preferably sent to recycling centers if possible, as recycling is a more environmentally friendly method. As part of the initial solution, the locations of the centers are determined randomly. A new center cannot be opened unless the capacity of the currently opened center is full. Waste residues are directed to disposal centers with appropriate capacities and minimum distances. This process is repeated for each waste type until all the waste generated over the planning horizon is collected.

\subsubsection{List of Operators}

In the proposed BOALNS, eight destroy and two insertion operators are used. After each insertion operator, we apply a repair procedure to generate feasible solutions. In the following, we describe these operators.

Destroy Operators We apply a total of eight destroy operators, the first three of which change the location configuration of centers $(\mathrm{O} 1-\mathrm{O} 3)$, while others change the visiting schedules of generation nodes (O4-O8). In each iteration, operators $\mathrm{O} 4$ to $\mathrm{O} 8$ are applied separately to each type of waste, as the vehicles cannot transport different types of waste at the same time considering the contamination risk of waste. These operators remove several $s$ generation nodes from their current position on a given route and insert them to the removal list, where $s$ is a uniform number between one and $S$. This route is the route with the highest idle vehicle capacity on a given day. The day is selected using the roulette wheel selection principle, and its probability is proportional to the total amount of waste generated that day. We note that the amount of waste collected from the source node per visit 


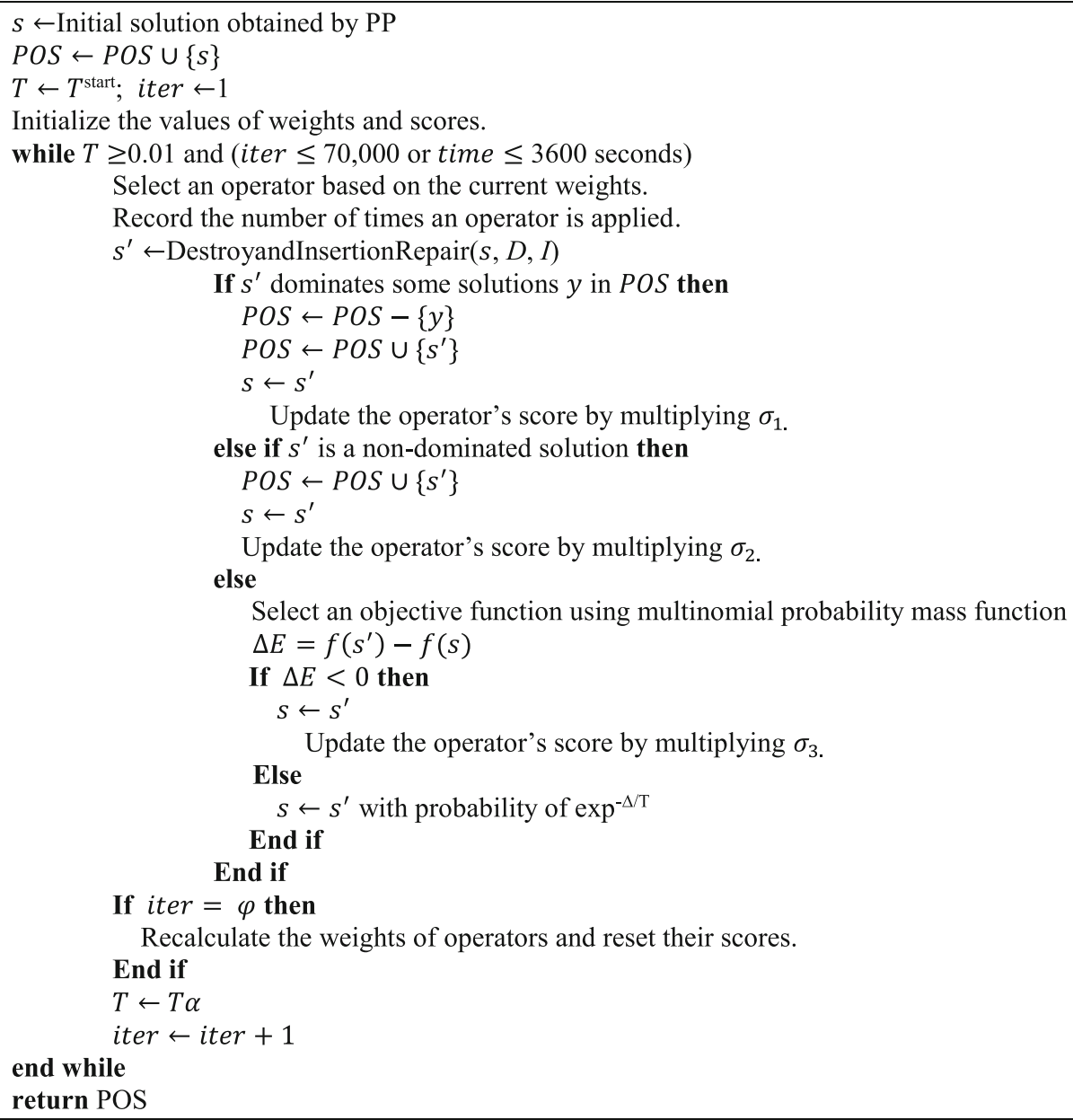

Fig. 2 Steps of BOALNS

obtained with PP is preserved throughout BOALNS iterations. It means that if a generation node is removed from a route, the amount collected from that node is offloaded from the current route's load and added to the load of the newly assigned route. If this yields to an infeasible solution, then the excess amount is assigned to the other visit of the generation node. The following describes the destroy operators used in BOALNS.

O1 Close Center: This operator randomly selects and closes one of the centers with minimum load. All nodes associated with the closed center are added to the removal list.

O2 Open Center: This operator opens a new center in a random location. The nodes that are closest to this center are removed from their routes.
O3 Swap Center: This operator closes a treatment center and opens a recycling center instead, as recycling is a more viable option according to the waste hierarchy. All nodes assigned to the closed center are added to the removal list.

O4 Randomly remove $s$ nodes: This operator randomly removes $s$ generation nodes.

O5 Randomly remove a route: This operator removes all nodes in a route.

O6 Worst distance $s$ nodes removal: Several generation nodes $s$ located at far distances are removed.

O7 Shaw removal: As suggested in Ropke and Pisinger [17] and Shaw [55], distance and demand are chosen as relatedness measures. This operator removes several waste generation nodes with similar distances and amounts of waste generated.

O8 Worst node removal: This operator removes a generation node that improves the objective function the most. 


\section{Insertion Operators}

O9 Greedy insertion: Greedy insertion operator is used in our algorithm, as suggested by Ropke and Pisinger [17]. This operator iteratively adds all nodes in the removal list one by one to the vehicle route's best possible position in a period that provides the least cost increase in the objective function.

O10 Random insertion: This operator inserts all nodes in the removal list to random positions.

Repair Procedures Destroy/insertion operators may change the sequence of nodes in a route, generate new routes, or assign new visit day combinations to generation nodes. When node $s$ is removed from the route on day $p$ by destroy operators, insertion operators may insert the node $s$ to the same route in a different position, to a route of another vehicle on the same day or to another route on a different day $p^{\prime}$. These changes may require checking the feasibility of the solution and recalculating the values of some affected decision variables. In these three cases, the repair procedures aimed at generating feasible solutions are as follows:

R1 Insert to the same route: Only the visit sequence of the generation nodes is changed. The vehicle's load after visiting node $s$ must be updated.

R2 Insert to a route of another vehicle on the same day: The visit days of the nodes are unchanged. The idle vehicle capacity of the newly assigned route must be greater than the amount of waste associated with node $s$. Amounts to be collected on this route, idle vehicle capacity, and the number of vehicles must be updated.

R3 Insert to another route on a different day $p$ ': The idle vehicle capacity on this route must be greater than the additional amount collected from node $s$. As visiting days of generation nodes change, their inventory levels must be recalculated. If the removed node $s$ is already a part of the route on day $p$ ', the amount of waste collected from node $s$ on day $p$ is updated by taking the total amount collected from node $s$ on days $p$ and $p$ '.

\section{Computational Experiments}

This section provides the details of the experimental study and discussions on the numerical results for BOALNS. The pick-up plan generation was solved via CPLEX 12.6, and BOALNS was coded in C ++ with Microsoft Visual Studio 2019 version 16.7.4. Experiments were run on a computer equipped with an Intel Core i7-6500U CPU, $2.5 \mathrm{GHz}$, and 8 GB RAM.
Table 2 Problem characteristics

\begin{tabular}{lllll}
\hline Pr. No & \multicolumn{2}{l}{ Number of Nodes } & \\
\cline { 2 - 5 } & Generation & Recycling & Treatment & Disposal \\
\hline 1 & 6 & 4 & 3 & 4 \\
2 & 8 & 5 & 4 & 4 \\
3 & 10 & 6 & 4 & 5 \\
4 & 12 & 7 & 5 & 6 \\
5 & 15 & 8 & 5 & 7 \\
6 & 20 & 8 & 6 & 7 \\
7 & 20 & 9 & 7 & 8 \\
8 & 25 & 10 & 7 & 9 \\
9 & 25 & 10 & 8 & 9 \\
10 & 30 & 11 & 8 & 10 \\
11 & 40 & 13 & 10 & 11 \\
12 & 50 & 16 & 11 & 14 \\
13 & 75 & 17 & 12 & 15 \\
14 & 90 & 20 & 13 & 17 \\
15 & 100 & 24 & 14 & 20 \\
\hline
\end{tabular}

Since PLIRP in waste management is first introduced in this paper, no solution has been reported in the literature for comparison. We derived benchmarking problems from the dataset presented by Rabbani et al. [35] for industrial waste location routing problems. As seen in Table 2, we generate 15 problem instances consisting of different numbers of generation nodes and centers. In contrast to Rabbani et al. [35], we assume that there are no existing (already established) centers in the system, which increases the number of potential centers, i.e., the size of the problem. Our dataset includes two different types of healthcare waste. There are two heterogeneous fleets of vehicles, each compatible with a single type of waste. Vehicle capacities are set to 200. The number of periods in a cycle is fixed to 5 days. We divided the total amount generated from each type of waste in each generation node by five to get the amount of waste type $w$ generated in period $p$ at node $s\left(M G_{s}^{w p}\right)$. In all instances, the storage capacity of the generation nodes and the inventory holding cost per period are set to 200 and 2, respectively. The mass loss rates for incineration and recycling centers are $80 \%$ and $60 \%$. A unit vehicle operating cost per period $(C O)$ is 2 . While the variable costs of treating each unit of waste with sterilization and incineration technologies are 5 and 7, they are 4 for recycling and disposal centers. All remaining data were taken following the framework described in Sect. 3.

Table 3 demonstrates the parameters and their values used in the BOALNS. These values were decided after numerous preliminary experiments. The temperature in the next iteration is obtained by reducing the current temperature $T_{i}$ in $i$ th iteration by the cooling ratio $(\alpha)$. The temperature $\left(T^{\text {start }}\right)$ starts at 30,000 and may drop to $0.01\left(T^{f i n a l}\right)$. The algo- 
Table 3 Parameters used in the BOALNS

\begin{tabular}{ll}
\hline Parameter & Value \\
\hline$\varphi$ & 200 \\
$T^{\text {start }}$ & 30,000 \\
$T^{\text {final }}$ & 0.01 \\
$\alpha$ & 0.99 \\
$\eta$ & 0.7 \\
$\sigma_{1}$ & 5 \\
$\sigma_{2}$ & 8 \\
$\sigma_{3}$ & 3 \\
\hline
\end{tabular}

rithm terminates when the temperature reaches its minimum value and when the iteration number or CPU time reaches its predetermined value. BOALNS performs well in CPU time since it finds solutions in less than 15 min for the largest-sized problem with 100 source nodes. The runtime of generating the pick-up plan solved by CPLEX is negligible with $7.06 \mathrm{~s}$, even for the $15^{\text {th }}$ problem example.

\subsection{Analysis of the BOALNS Algorithm}

We first analyze the success of each operator in terms of its percentage usage frequency during the search. Table 4 provides the results for some selected problems in different sizes. The analysis shows that the success of the operator varies between problem instances. The frequency of using the destroy operators of $\mathrm{O} 3$ and $\mathrm{O} 8$ and the insertion operator of $\mathrm{O} 9$ is, on average, higher than the use of other operators in BOALNS. O1 and O5 are the least critical operators with the lowest average percentage of success. From this analysis, we conclude that $\mathrm{O} 3, \mathrm{O} 8$, and $\mathrm{O} 9$ significantly impact the solution quality of the algorithm.

We then compare the results of BOALNS with those provided by the Adaptive Large Neighborhood Search Algorithm (ALNS). At each iteration, ALNS evaluates each solution based on Eq. (35), which is a scalar composite function of the weighted sum of the objectives.

$\theta(x)=w_{1} \theta_{1}(x)+w_{2} \theta_{2}(x)+\cdots+w_{n} \theta_{n}(x)$
Table 5 Comparison of ALNS with BOALNS

\begin{tabular}{lll}
\hline Prob No & \multicolumn{2}{l}{ Dev $(\%)$} \\
\cline { 2 - 3 } & Cost & Risk \\
\hline 1 & 0.56 & 17.19 \\
2 & 3.39 & 21.72 \\
3 & 4.1 & 23.1 \\
4 & 6.9 & 25 \\
5 & 8.25 & 32.9 \\
6 & 17.3 & 29.76 \\
7 & 12.53 & 36 \\
8 & 12.61 & 39.34 \\
9 & 18.36 & 42.2 \\
10 & 22.77 & 40 \\
11 & 31.04 & 43.42 \\
12 & 26.96 & 45.94 \\
13 & 39.05 & 50.07 \\
14 & 39.6 & 52.8 \\
15 & 40.2 & 51.74 \\
Average & 18.91 & 36.75 \\
\hline
\end{tabular}

where $w_{i}$ is a positive constant weight associated with the function $\theta_{i}(x)$ and is randomly determined in each iteration of the algorithm $\left(\sum_{i=1}^{n} w_{i}=1\right)$.

We note that ALNS is not able to generate Pareto-optimal solutions. Therefore, to fairly compare these algorithms, we found the average values of the Pareto-optimal solutions obtained with BOALNS for each problem in terms of both objective functions. Table 5 presents the percentage deviation of the solution obtained by the weighted sum approach from the one provided with the multinomial probability mass function for all problem instances. In other words, it refers to the percent deterioration in solution quality of ALNS compared to BOALNS. The average deviations for both cost and risk objectives are less than $37 \%$. This comparison shows that the BOALNS is consistently superior to ALNS in all instances. Furthermore, it is observed that as the problem size increases, the percentage deviation from the mean of the objective values of the Pareto-optimal solutions also increases.

We then evaluate the performance of BOALNS by comparing it with the Bi-Objective Large Neighborhood Search
Table 4 Percentage frequency of using operators

\begin{tabular}{lllllllllll}
\hline Pr. No & O1 & O2 & O3 & O4 & O5 & O6 & O7 & O8 & O9 & O10 \\
\hline 1 & 15 & 21 & 15 & 18 & 15 & 22 & 24 & 24 & 32 & 22 \\
4 & 15 & 17 & 18 & 19 & 15 & 22 & 18 & 28 & 35 & 21 \\
7 & 13 & 17 & 29 & 19 & 17 & 21 & 20 & 27 & 36 & 24 \\
10 & 20 & 18 & 29 & 23 & 16 & 23 & 16 & 26 & 32 & 25 \\
12 & 18 & 22 & 26 & 22 & 15 & 21 & 23 & 30 & 30 & 20 \\
15 & 18 & 15 & 34 & 20 & 14 & 25 & 18 & 32 & 37 & 21 \\
Avg & 16.50 & 18.33 & 25.17 & 20.17 & 15.33 & 22.33 & 19.83 & 27.83 & 33.67 & 22.17 \\
\hline
\end{tabular}


Table 6 Comparison between BOALNS and BOLNS

\begin{tabular}{llllll}
\hline Prob No & \multicolumn{2}{l}{ NPS } & & \multicolumn{2}{l}{ Percent of Domination } \\
\cline { 2 - 3 } \cline { 5 - 6 } & BOALNS & BOLNS & & BOALNS & BOLNS \\
\hline 1 & 4 & 3 & & 0.50 & 0 \\
2 & 6 & 5 & 0.66 & 0.6 \\
3 & 9 & 6 & & 0.77 & 0.50 \\
4 & 10 & 7 & 0.80 & 0.71 \\
5 & 11 & 9 & 0.63 & 0.55 \\
6 & 13 & 11 & 0.69 & 0.44 \\
7 & 12 & 10 & & 0.83 & 0.5 \\
8 & 12 & 8 & & 0.72 & 0.42 \\
9 & 14 & 10 & & 0.92 & 0.7 \\
10 & 15 & 12 & & 0.66 & 0.58 \\
11 & 11 & 7 & 0.82 & 0.67 \\
12 & 17 & 12 & 0.82 & 0.58 \\
13 & 16 & 13 & 0.78 & 0.31 \\
14 & 15 & 13 & 0.88 & 0.71 \\
15 & 17 & 14 & 0.76 & 0.73 \\
Average & 12.13 & 9.33 & 0.75 & 0.53 \\
\hline
\end{tabular}

Algorithm (BOLNS), which employs the bi-objective structure given in Fig. 2 and neglects the past success of the operators. We used two metrics in evaluating the performances of these two algorithms. The first is the average number of Pareto-optimal solutions (NPS), and the second is the percent domination calculated by Eq. (36). The Pareto- optimal solutions from both algorithms are aligned together to form the $S$ set (i.e., $S=S_{1} \cup S_{2}$ ). Dominated solutions of an algorithm are then removed from the set $S$ to calculate the percentage domination.

$R_{\text {pos }}\left(S_{i}\right)=\frac{\left|S_{i}-\left\{X \in S_{i} \mid \exists Y \in S: Y \prec X\right\}\right|}{\left|S_{i}\right|}$

Table 6 shows these two metrics' values for ten runs of the BOALNS and BOLNS algorithms. BOALNS is able to generate relatively more Pareto-optimal solutions than BOLNS. It also outperforms BOLNS based on the percent domination in all problems. For example, the zero value for $R_{P O S}\left(S_{2}\right)$ in the first problem instance indicates that the solutions obtained with BOALNS dominate all solutions generated by BOLNS. While the percent domination in BOALNS varies from 50 to $92 \%$, it changes between 0 and $71 \%$ in BOLNS. These results indicate that using the adaptive search engine enhances BOALNS' ability to reach high-quality Paretooptimal solutions. This is because BOLNS explores a new neighborhood with one randomly selected operator, while the other explores with the one chosen based on its past success.

We also apply our algorithm to the traditional LRP. BOALNS is flexible as it solves LRP without any changes to the algorithm. Pareto-optimal solutions of problems 1 to 10 and 11 to 15 obtained for both PLIRP and LRP are given in Fig. 3. As expected, the Pareto-optimal solutions obtained for PLIRP are worse than those obtained for LRP in terms of the risk objective, as PLIRP allows multiple visits to customers. However, in most cases, PLIRP generates higher quality solu-
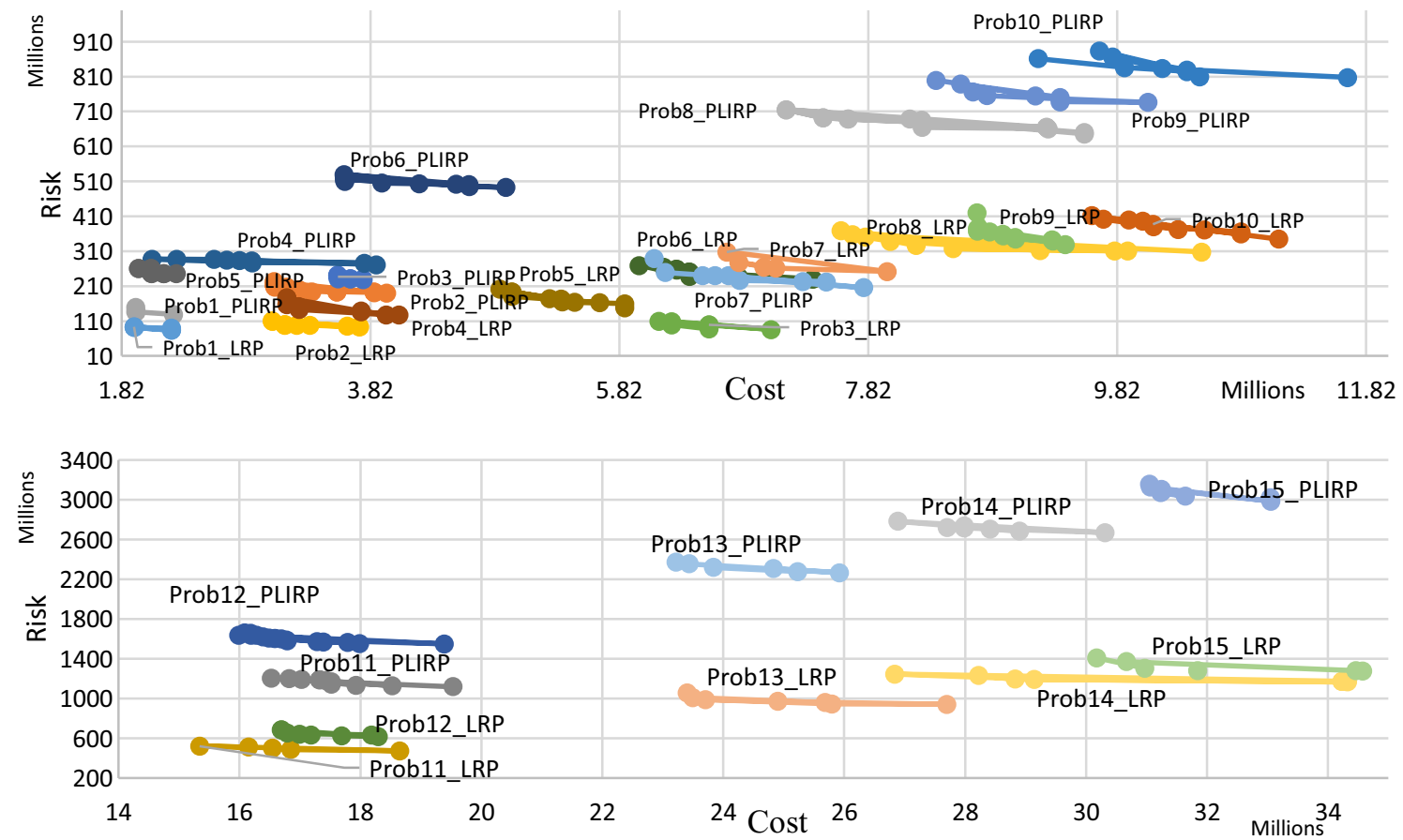

Fig. 3 Pareto-optimal solutions of LRP and PLIRP for instances between 
tions than LRP in terms of cost objective. This analysis shows practitioners that for realistic problems where periodic collection, inventory, and location selection decisions are taken together, as suggested in PLIRP, many high-quality solutions can be achieved with little computational effort in terms of cost objective.

We conclude that the proposed solution approach is practical and can be used to plan and develop real-life waste management systems. Furthermore, all participants in the waste management system can benefit from the results of the proposed study.

(1) The most crucial information obtained from the decision maker's point of view is the locations of the waste processing centers, the visit schedules to the waste producers, and the treatment technologies to be established. Pareto solutions offer comprehensive selections for these decisions.

(2) The proposed bi-level solution approach allows decision makers to design a waste management system that simultaneously minimizes the operating cost of the network and reduces the risk of virus contamination through waste.

(3) Decision makers can estimate the cost of the waste management system and the overall risk associated with the transport of healthcare waste and the location of centers. They can prepare regional and provincial management strategies and finalize investment needs. They can also optimize the number of people exposed to waste during transportation and site selection, taking into account the risk to human health of contaminated waste.

(4) In the event of unexpected increases in healthcare waste, such as during the COVID-19 pandemic, emerging needs must be responded to promptly. The proposed solution method provides quick information on the number of vehicles needed and the visit schedules to healthcare facilities for managers responsible for the timely and appropriate waste collection.

(5) Healthcare facilities will have known waste collection programs. Thus, they can benefit from the results by arranging their waste handling activities according to visiting schedules, if possible. They can reduce the amount of hazardous waste by effectively separating hazardous and non-hazardous wastes. Those with insufficient storage capacities can use on-site incineration. Managers can provide mobile incineration or sterilization systems or increase the frequency of visits to deal with excessive waste. The periodic waste collection helps minimize the inventory holding cost, the risk of contamination, and the spread of the virus through waste.

(6) The results encourage waste treatment centers to comply with legal regulations according to capacity and needs.

\section{Conclusion}

This study was motivated by the challenges faced by countries in processing and disposing of increasing amounts of healthcare waste during the COVID-19 pandemic. Scientific articles and the World Health Organization (WHO) point out that improper disposal of waste can cause further spread of the virus. Therefore, systems must focus on safe and appropriate treatment technologies, increase their waste handling capacity and frequency of waste collection to prevent this threat. To the best of our knowledge, no study in the literature develops a theoretical or practical approach for collecting periodic healthcare waste considering the storage capacities of facilities and establishing of recycling, treatment, and disposal centers. In order to fill the gap in the literature, the study aims to design sustainable and effective waste collection and treatment networks by optimizing operational and strategic decisions simultaneously. It presents a bi-objective MINLP formulation that minimizes the transportation costs between nodes, fixed location and variable costs at centers, the total cost of holding inventory at source nodes, and the total risk associated with transporting waste and locating centers. By including existing centers in the model formulation, decision makers can analyze the required increase in capacities and the number of vehicles and facilities in the current system. Furthermore, the proposed network representation and the developed model can be applied to other real-life problems such as second-hand goods collection, mailbox collection, and blood collection that require periodic collection of several different items and transporting then to their respective centers for processing.

To cope with the complexity of the proposed problem, we offer a two-step solution approach. The first stage consists of solving a mixed-integer linear model, which finds the visiting schedules and waste amounts collected from generation nodes. The second stage presents a BOALNS that deals with vehicle routes, locations of centers, and transportation of waste and waste residues. Our algorithm generates Paretooptimal solutions and uses several destroy and insertion operators, adaptive search mechanism, simulated annealing criteria, and multinomial probability mass function. The performance of BOALNS was compared with ALNS and BOLNS. Experimental results showed that BOALNS outperforms these algorithms based on several performance evaluation metrics. In addition, we observed that the adaptive search engine increases the capability of BOALNS to achieve high-quality Pareto-optimal solutions. Moreover, the performance of BOALNS is investigated in generating Pareto-optimal solutions for location routing problems. We showed that the proposed BOALNS is flexible as it solves location routing problems without any changes to the algorithm and obtains many high-quality solutions with little computational effort in terms of cost objective. 
As the problem and the solution approach proposed in this study are newly introduced, they still have expansion potential. In future, the performance of the BOALNS can be improved by implementing some other mechanisms such as mutation and tabu list. In addition, the amount of waste at the source nodes can be considered stochastic, and new solution methods can be proposed accordingly. Temporary storage facilities can be established to cope with increasing waste, especially during the COVID-19 period. Contaminated waste from quarantine houses can also be included in the system. Some part of the capacity of recycling centers can be allocated for household waste. Finally, developing a decision support system can be helpful to decision makers, especially when making operational decisions.

\section{References}

1. WHO: Health-care Waste. https://www.who.int/news-room/factsheets/detail/health-care-waste (2018). Accessed 23 June 2021

2. Ilyas, S.; Srivastava, R.R.; Kim, H.: Disinfection technology and strategies for COVID-19 hospital and bio-medical waste management. Sci. Total Environ. 749, 141652 (2020). https://doi.org/10.1 016/j.scitotenv.2020.141652

3. European Union: Directive 2008/98/EC of the European Parliament and the Council on waste. https://eur-lex.europa. eu/LexUriServ/LexUriServ.do?uri=OJ:L:2008:312:0003:00 30:en:PDF\#: :text=This\%20Directive\%20lays $\% 20$ down $\% 2$ 0measures,the $\% 20$ efficiency $\% 20$ of $\% 20$ such $\% 20$ use (2008). Accessed 23 June 2021

4. Das, A.K.; Islam, M.N.; Billah, M.M.; Sarker, A.: Review COVID19 pandemic and health care solid waste management strategy A mini-review. Sci. Total Environ. 778, 146220 (2021). https://doi. org/10.1016/j.scitotenv.2021.146220

5. Hantoko, D.; Li, X.; Pariatamby, A.; Yoshikawa, K.; Horttanainen, M.; Yan, M.: Challenges and practices on waste management and disposal during COVID-19 pandemic. J. Environ. Manage. 286, 112140 (2021). https://doi.org/10.1016/j.jenvman.2021.112140

6. Nzediegwu, C.; Chang, S.X.: Improper solid waste management increases potential for COVID-19 spread in developing countries. Resour. Conserv. Recycl. 161, 104947 (2020). https://doi.org/10.1 016/j.resconrec.2020.104947

7. Sharma, H.B.; Vanapalli, K.R.; Cheela, V.R.S.; Ranjan, V.P.; Jaglan, A.K.; Dubey, B.; Goal, S.; Bhattacharya, J.: Challenges, opportunities, and innovations for effective solid waste management during and post COVID-19 pandemic. Resour. Conserv. Recycl. 162, 105052 (2020). https://doi.org/10.1016/j.resconrec. 2020.105052

8. Perl, J.; Daskin, M.S.: A warehouse location-routing problem. Transp. Res. Part B Methodol. 19(5), 381-396 (1985). https://doi. org/10.1016/0191-2615(85)90052-9

9. Rifai, A.P.; Nguyen, H.; Dawal, S.ZMd.: Multi-objective adaptive large neighborhood search for distributed reentrant permutation flow shop scheduling. Appl. Soft Comput. 40, 42-57 (2016). https://doi.org/10.1016/j.asoc.2015.11.034

10. Aksen, D.; Kaya, O.; Salman, F.S.; Tuncel, O.: An adaptive large neighborhood search algorithm for a selective and periodic inventory routing problem. Eur. J. Oper. Res. 239(2), 413-426 (2014). https://doi.org/10.1016/j.ejor.2014.05.043

11. Cardenas-Barron, L.E.; Gonzalez-Velarde, J.L.; Trevino-Garza, G.; Garza-Nunez, D.: Heuristic algorithm based on reduce and optimize approach for a selective and periodic inventory routing problem in a waste vegetable oil collection environment. Int. J. Prod. Econ. 211, 44-59 (2019). https://doi.org/10.1016/j.ijpe.201 9.01.026

12. Coelho, L.C.; Cordeau, J.-F.; Laporte, G.: The inventoryrouting problem with transshipment. Comput. Oper. Res. 39(11), 2537-2548 (2012). https://doi.org/10.1016/j.cor.2011.12.020

13. Hemmelmayr, V.C.; Cordeau, J.-F.; Crainic, T.G.: An adaptive large neighborhood search heuristic for two-echelon vehicle routing problems arising in city logistics. Comput. Oper. Res. 39(12), 3215-3228 (2012). https://doi.org/10.1016/j.cor.2012.04.007

14. Hemmelmayr, V.C.: Sequential and parallel large neighborhood search algorithms for the periodic location routing problem. Eur. J. Oper. Res. 243, 52-60 (2015). https://doi.org/10.1016/j.ejor.201 4.11.024

15. Koç, Ç.; Bektas, T.; Jabali, O.; Laporte, G.: The fleet size and mix pollution-routing problem. Transp. Res. Part B Methodol. 70, 239-254 (2014). https://doi.org/10.1016/j.trb.2014.09.008

16. Koç, Ç.: A unified-adaptive large neighborhood search metaheuristic for periodic location-routing problems. Transp. Res. Part C 68, 265-284 (2016). https://doi.org/10.1016/j.trc.2016.04.013

17. Ropke, S.; Pisinger, D.: An adaptive large neighborhood search heuristic for the pickup and delivery problem with time windows. Transp. Sci. 40(4), 455-472 (2006). https://doi.org/10.1287/trsc.1 050.0135

18. Pisinger, D., Ropke, S.: Handbook of metaheuristics (2nd ed.). In: Glover, F.W., Kochenberger, G. A. (eds.) Large neighborhood search Springer, pp. 399-419. Springer, New York (2010)

19. Aboutahoun, A.W.: Combined distance-reliability model for hazardous waste transportation and disposal. Life Sci. J. 9(2), 1286-1295 (2012)

20. Alumur, S.; Kara, B.Y.: A new model for the hazardous waste location-routing problem. Comput. Oper. Res. 34(5), 1406-1423 (2007). https://doi.org/10.1016/j.cor.2005.06.012

21. Aydemir-Karadag, A.: A profit-oriented mathematical model for hazardous waste locating routing problem. J. Clean. Prod. 202, 213-225 (2018). https://doi.org/10.1016/j.jclepro.2018.08.106

22. Boyer, O.; Hong, T.S.; Pedram, A.; Yusuff, R.B.; M., Zulkifli, N. : A Mathematical model for the industrial hazardous waste locationrouting problem. J. Appl. Math. (2013). https://doi.org/10.1155/2 $013 / 435272$

23. Current, J.; Ratick, S.: A model to assess risk, equity and efficiency in facility location and transportation of hazardous materials. Locat. Sci. 3(3), 187-201 (1995). https://doi.org/10.1016/0966-8349(95 )00013-5

24. Emek, E.; Kara, B.Y.: Hazardous waste management problem: the case for incineration. Comput. Oper. Res. 34(5), 1424-1441 (2007). https://doi.org/10.1016/j.cor.2005.06.011

25. Giannikos, I.: A multi-objective programming model for locating treatment sites and routing hazardous wastes. Eur. J. Oper. Res. 104(2), 333-342 (1998). https://doi.org/10.1016/S0377-2217(97)0 0188-4

26. Nema, A.K.; Gupta, S.K.: Optimization of regional hazardous waste management systems: an improved formulation. Waste Manage. 19(7-8), 441-451 (1999). https://doi.org/10.1016/S0956-053 $\mathrm{X}(99) 00241-\mathrm{X}$

27. Samanlioglu, F.: A multi-objective mathematical model for the industrial hazardous waste location-routing problem. Eur. J. Oper. Res. 226(2), 332-340 (2013). https://doi.org/10.1016/j.ejor.2012. 11.019

28. Xie, Y.; Lu, W.; Wang, W.; Quadrifoglio, L.: A multimodal location and routing model for hazardous materials transportation. J. Hazard. Mater. 227-228, 135-141 (2012). https://doi.org/10.1016/ j.jhazmat.2012.05.028

29. Yilmaz, O.; Kara, B.Y.; Yetis, U.: Hazardous waste management system design under population and environmental impact consid- 
erations. J. Environ. Manage. 203(2), 720-731 (2017). https://doi. org/10.1016/j.jenvman.2016.06.015

30. Zografos, K.G.; Samara, S.: Combined location-routing model for hazardous waste transportation and disposal. Transp. Res. Rec. 1245, 52-59 (1989)

31. Yu, H.; Sun, X.; Solvang, W.D.; Zhao, X.: Reverse logistics network design for effective management of medical waste in epidemic outbreaks: insights from the coronavirus disease 2019 (COVID19) outbreak in Wuhan (China). Int. J. Environ. Res. Public Health 17(5), 1770 (2020). https://doi.org/10.3390/ijerph17051770

32. Kargar, S.; Pourmehdi, M.; Paydar, M.M.: Reverse logistics network design for medical waste management in the epidemic outbreak of the novel coronavirus (COVID-19). Sci. Total Environ. 746, 141183 (2020). https://doi.org/10.1016/j.scitotenv.2020. 141183

33. Zhao, J.; Verter, V.: A bi-objective model for the used oil locationrouting problem. Comput. Oper. Res. 62, 157-168 (2015). https:// doi.org/10.1016/j.cor.2014.10.016

34. Zhao, J.; Ke, G.Y.: Incorporating inventory risks in location-routing models for explosive waste management. Int. J. Prod. Econ. 193, 123-136 (2017). https://doi.org/10.1016/j.ijpe.2017.07.001

35. Rabbani, M.; Heidari, R.; Farrokhi-Asl, H.; Rahimi, N.: Using metaheuristic algorithms to solve a multi-objective industrial hazardous waste location-routing problem incompatible waste types. J. Clean. Prod. 170, 227-241 (2018). https://doi.org/10.1016/j. jclepro.2017.09.029

36. Rabbani, M.; Heidari, R.; Yazdanparast, R.: A stochastic multiperiod industrial hazardous waste location-routing problem: integrating NSGA-II and Monte Carlo simulation. Eur. J. Oper. Res. 272(3), 945-961 (2019). https://doi.org/10.1016/j.ejor.2018.07.02 4

37. Farrokhi-Asl, H.; Makui, A.; Jabbarzadeh, A.; Barzinpour, F.: Solving a multi-objective sustainable waste collection problem considering a new collection network. Oper. Res. Int. J. 20, 1977-2015 (2020). https://doi.org/10.1007/s12351-018-0415-0

38. Nikzamir, M.; Baradaran, V.: A healthcare logistic network considering stochastic emission of contamination: bi-objective model and solution algorithm. Transp. Res. Part E 142, 102060 (2020). https://doi.org/10.1016/j.tre.2020.102060

39. Tirkolaee, E.B.; Abbasian, P.; Weber, G.W.: Sustainable fuzzy multi-trip location-routing problem for medical waste management during the COVID-19 outbreak. Sci. Total Environ. 756, 143607 (2021). https://doi.org/10.1016/j.scitotenv.2020.143607

40. Campbell, A.M.; Wilson, J.H.: Forty years of periodic vehicle routing. Networks 63(1), 2-15 (2014). https://doi.org/10.1002/net.215 44

41. Beltrami, E.J.; Bodin, L.D.: Networks and vehicle routing for municipal waste collection. Networks 4(1), 65-94 (1974). https:// doi.org/10.1002/net.3230040106
42. Russell, R.; Igo, W.: An assignment routing problem. Networks 9(1), 1-17 (1979). https://doi.org/10.1002/net.3230090102

43. Christofides, N.; Beasley, J.E.: The period routing problem. Networks 14(2), 237-256 (1984). https://doi.org/10.1002/net.323014 0205

44. Archetti, C.; Fernández, E.; Huerta-Muñoz, D.L.: The flexible periodic vehicle routing problem. Comput. Oper. Res. 85, 58-70 (2017). https://doi.org/10.1016/j.cor.2017.03.008

45. Andersson, H.; Hoff, A.; Christiansen, M.; Hasle, G.; Lokketangen, A.: Industrial aspects and literature survey: combined inventory management and routing. Comput. Op. Res. 37(9), 1515-1536 (2010). https://doi.org/10.1016/j.cor.2009.11.009

46. Bertazzi, L.; Speranza, M.: Inventory routing problems: an introduction. Euro J. Transp. Logist. 1, 307-326 (2012). https://doi.org/ 10.1007/s13676-012-0016-7

47. Bertazzi, L.; Speranza, M.: Inventory routing problems with multiple customers. Euro J. Transp. Logist. 2, 255-275 (2013). https:// doi.org/10.1007/s13676-013-0027-z

48. Coelho, L.C.; Cordeau, J.-F.; Laporte, G.: Thirty years of inventory routing. Transp. Sci. 48(1), 1-19 (2013). https://doi.org/10.1287/ trsc.2013.0472

49. Aksen, D.; Kaya, O.; Salman, F.S.; Akca, Y.: Selective and periodic inventory routing problem for waste vegetable oil collection. Optim. Lett. 6(6), 1063-1080 (2012). https://doi.org/10.1007/s11 590-012-0444-1

50. Taslimi, M.; Batta, R.; Kwon, C.: Medical waste collection considering transportation and storage risk. Comput. Oper. Res. 120, 104966 (2020). https://doi.org/10.1016/j.cor.2020.104966

51. Prodhon, C.: An iterative metaheuristic for the periodic locationrouting problem. In: Nickel, S.; Kalcsics, J. (Eds.) Operations Research Proceedings, pp. 159-164. Springer, Heidelberg (2007)

52. Prodhon, C.: A hybrid evolutionary algorithm for the periodic location-routing problem. Eur. J. Oper. Res. 210(2), 204-212 (2011). https://doi.org/10.1016/j.ejor.2010.09.021

53. Kulturel-Konak, S.; Smith, A.E.; Norman, B.: Multi-objective tabu search using a multinomial probability mass function. Eur. J. Oper. Res. 169(3), 918-931 (2006). https://doi.org/10.1016/j.ejor.2004. 08.026

54. Cakir, B.; Altıparmak, F.; Dengiz, B.: Multi-objective optimization of a stochastic assembly line balancing: a hybrid simulated annealing algorithm. Comput. Ind. Eng. 60(3), 376-384 (2011). https:// doi.org/10.1016/j.cie.2010.08.013

55. Shaw, P.: Using constraint programming and local search methods to solve vehicle routing problems. In: Maher, M.; Puget, J.F. (Eds.) Principles and Practice of Constraint Programming- CP98, 1520, pp. 417-431. Springer, Heidelberg (1998) 